\title{
Co-occurrence of Babesia microti, Bartonella spp., Borrelia burgdorferi s.l. and Anaplasma phagocytophilum in rodents from Lower Silesia, Poland
}

\author{
J Hildebrand, K Buńkowska-Gawlik, A Perec-Matysiak \\ From The 1st Conference on Neglected Vectors and Vector-Borne Diseases (EurNegVec): with Management \\ Committee and Working Group Meetings of the COST Action TD1303 \\ Cluj-Napoca, Romania. 8-11 April 2014
}

This study aims to establish the relative contribution of rodent populations from diverse habitats to the occurrence of rodent-borne pathogens of public health significance. Rodents $(\mathrm{n}=492)$ represented by Apodemus agrarius, A. flavicollis and Myodes glareolus, were captured in live traps in four localities of south-western Poland (20092012). For the analysis of co-occurrence of pathogens, Babesia microti, Borrelia burgdorferi s.l., Bartonella spp. and Anaplasma phagocytophilum, both blood and spleen samples were obtained from selected rodent specimens. The choice of genetic markers and primers was based on the literature data and our preliminary results. Conventional PCR was used for the detection of DNA of examined pathogens. Selected PCR positive products were purified and sequenced. BLAST searches were conducted in order to elucidate any homologies with previously deposited sequences in GenBank.

The DNA of pathogens was detected in $66.7 \%$ of the rodents tested. We observed that among infected rodents, $40.5 \%$ were infected with at least two pathogens, while only $4.7 \%$ with all four pathogens. All three of the rodent species were infected with each of the examined pathogens. In examined rodent populations the prevalence of B. microti was $40.0 \%$, Bartonella spp. $37.7 \%$, B. burgdorferi s.l. $28.2 \%$ and A. phagocytophilum $17.7 \%$.

In each of the tissue samples (blood or spleen), the prevalences of Babesia microti and Bartonella spp. were recorded as comparable, on rather high levels. Interestingly, the occurrence of these pathogens in both blood and spleen was only detected in $13 \%$ of the rodents.

While examining the blood and spleen-derived DNA, it was found that as many as $92.7 \%$ of $A$. agrarius harbored at least one pathogen. Co-occurrence of 3-4 pathogens was most common in this rodent species (32.1\% infected).

By examining the spleen and blood samples of rodents at the same time, we estimate that the prevalence of pathogens in these rodents is higher than the literature indicates. This would implicate the examined rodent species as a significant reservoir of pathogens with zoonotic potential. Additionally, the role of $A$. agrarius, now widespread in some regions of Europe, as a reservoir host needs to be emphasized. A. agrarius can act as a bridge between woodland habitats and periurban environments frequented by humans.

Published: 1 April 2014

doi:10.1186/1756-3305-7-S1-O4

Cite this article as: Hildebrand et al: Co-occurrence of Babesia microti,

Bartonella spp., Borrelia burgdorferi s.l. and Anaplasma phagocytophilum in rodents from Lower Silesia, Poland. Parasites \& Vectors 2014 7(Suppl 1):O4.

* Correspondence: agnieszka.perec-matysiak@microb.uni.wroc.pl

Department of Parasitology, University of Wrocław; Przybyszewskiego Str. 63, 51-148 Wrocław, Poland 